\title{
Assessment of capacity support and scattering in experimental high speed vehicle to vehicle MIMO links
}

\author{
Patrick C.F. Eggers, Tim W. C. Brown, Kim Olesen, Gert Frølund Pedersen \\ Antennas, Propagation and Radio Networking sect. \\ Dept. of Electronic Systems, Aalborg University, Denmark \\ Email: $\{$ pe, tbr, ko, gfp\}@kom.aau.dk
}

\begin{abstract}
Preliminary results on the use of the vehicle to vehicle MIMO channel in a rural highway environment are presented. This is looked at both in terms of the available spatial multiplexing through singular value decomposition and also angular distribution within the channel. Results indicate a strong predominant line of sight link in general while instances of scattering from other vehicles will cause changes in the Doppler spectrum as well as beamforming jitter.
\end{abstract}

\section{INTRODUCTION}

Vehicle to vehicle (VTV) communications are a newer addition to the palette of wireless communication scenarios. While the motor industry has been looking at traffic guidance solutions (e.g. traffic information, collision avoidance, parking aid etc.), professional transport, safety/distress operators have been looking at seamless operation on the move (e.g. fleet management, cargo control/monitoring, home facilities assessable at the spot of need etc.). It can also be foreseen that private (peer to peer) broadband VTV application can find a market too (such as teleconferencing in a convoy of vehicles, exploiting long haul transport time).

Multiple antenna (MIMO) systems are an important addition to nearly all modern wireless communication systems due to their potential for space domain control. This can be used either for sheer capacity and throughput increase for a single pair of users, like through launch of orthogonal parallel channels (SVD)[1][3][2] or to reduce interference effects through spatial filtering [4].

In case of long time convoy operation, stability of capacity/throughput is important. In case of opposing (incident) traffic conditions, MIMO links will only have a short lifetime, stressing importance of reliability at very high 'spot capacity/throughput'.

Previous experimental VTV investigations have either been narrowband and/or only SISO [6]. The experiments presented here seem to be the first reported fully simultaneous VTV MIMO investigations (in high speed scenarios). We have used the $3.5 \mathrm{GHz}$ band which is one of the candidate bands for $4 \mathrm{G}$ applications.

\section{MEASUREMENT SETUP}

We have used our own proprietary radio channel sounder [5], operated at $3.5 \mathrm{GHz}$ and capable of fully simultaneous 16x4 MIMO link sounding (in total 16x32 if additional switching is employed). The sounder uses code domain separation to isolate different transmitter links [5]. The simultaneous sounding of the full MIMO channel matrix constellation is essential in high speed applications (like the highway measurements presented here) to avoid the need to interpolate to align channels. We have used the full sounding bandwidth of $100 \mathrm{MHz}$ in the experiments. However, in this paper we will only focus on the narrow band aspects.

Two vans were each equipped with a roof mount 4 element uniform linear array (ULA), oriented perpendicular to the line of travel (See figures 1 and 2). The elements were printed monopoles separated by $0.4 \lambda$. Preceding Electromagnetic model simulations [7] revealed that galvanic (conducting cable) connection could be used without disturbing the array. A further measurement was conducted with a lap-top mount array placed inside the leading vehicle in the convoy setup (See Figure 2).

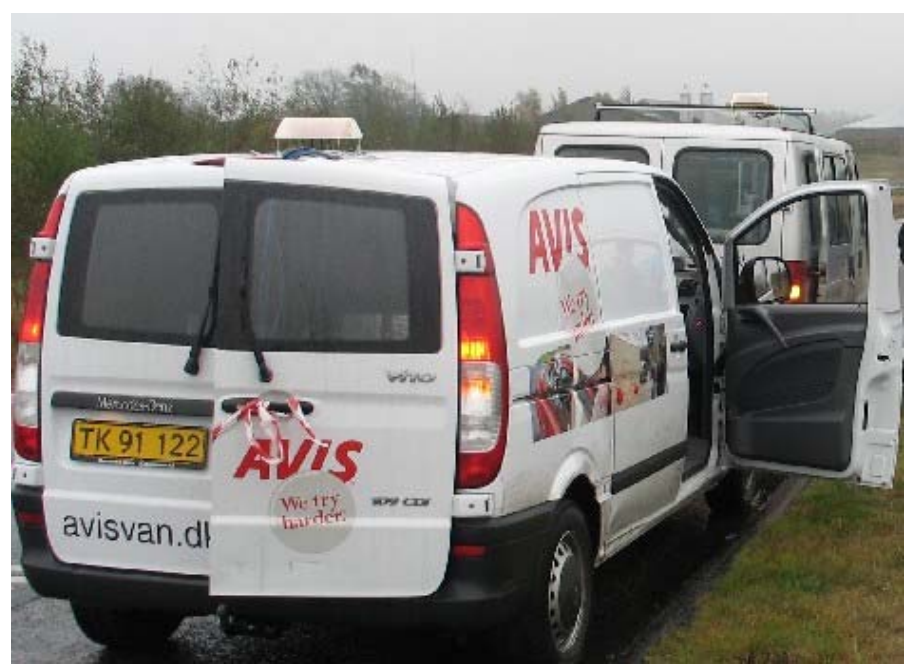

Fig. 1. Picture of the two vehicles with their respective linear array antennas

Due to exceedingly large amounts data for $4 \times 4$ links with 


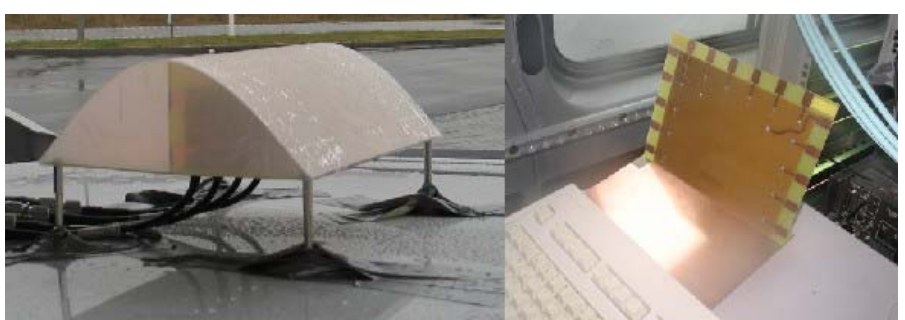

Fig. 2. Picture showing the rooftop and inside laptop antennas applied in the measurements

$100 \mathrm{MHz}$ bandwidth and $2540 \mathrm{~Hz}$ required sampling rate ${ }^{1}$, the measurement mode was partitioned in sections of $300 \mathrm{~ms}$ (175 sections, each stretching 10 meters) duration on-time, separated by $800 \mathrm{~ms}$ idle-time (allowing data transfer to the memory). Each section had 100 samples of the complete channel matrix.

\section{VTV HIGHWAY ENVIRONMENT}

The test area was a $1.1 \mathrm{~km}$ highway (E45) stretch in Northern Jutland, bordered by two bridges with nearby entries/exits that allowed easy access to the start and stop of the test track. The rural surroundings are typical for longer haul highways in Northern Europe. We distinguish between two modes: convoy where the vans trail each other with near to same speed and separation and opposing (incident) traffic, were the vans would pass each other while driving on their own respective sides (See figure 3).

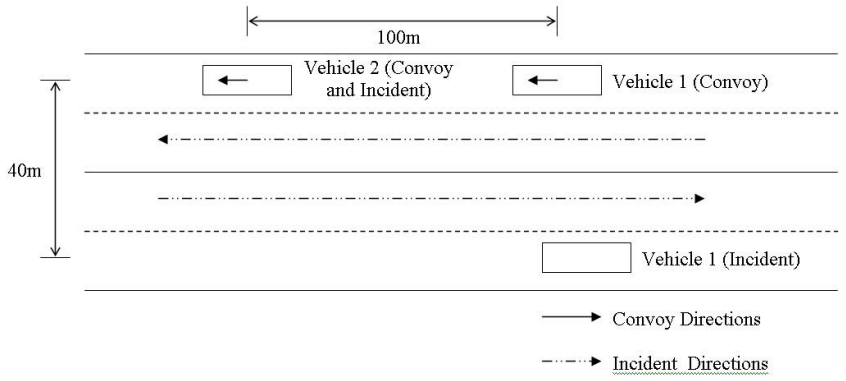

Fig. 3. Diagram of the vehicle measurement directions and how each vehicle was positioned

In convoy mode, the two vans would cruise with approx. $3 \mathrm{~s}$ delay between them at a measurement speed of $130 \mathrm{~km} / \mathrm{h}$. At this speed the typical separation between vans is $100 \mathrm{~m}$. Obstructing traffic in between the vehicles only happened occasionally in overtake situations, thus most of the time providing good line-of-sight (LOS) conditions between the two vehicles arrays. In the incident mode, the two vans were synchronized to pass each other in an open highway region where they each travelled $130 \mathrm{~km} / \mathrm{h}$ - and where the predicted

$1 \sim \lambda / 3.25$ at $130 \mathrm{~km} / \mathrm{h}$, satisfying the Nyquist criteria for a single moving terminal (and for two terminals with aligned movement as in the convoy scenario) but slightly insufficient for the dual moving terminals in a head to head incident traffic scenario crossing point on the highway could be repeated within around $\pm 100 \mathrm{~m}$.

\section{SCATTERING RICHNESS AND CAPACITY STABILITY}

The measured (narrowband) channel is denoted by $\mathbf{H}$ which is $N_{R X} \times N_{T X}(4 \mathrm{x} 4$ in our case) dimensional channel transfer matrix (incl. measurement noise). Each element $H_{i j}$ of the channel transfer matrix $\mathbf{H}$ corresponds to the gain from the $j$-th transmit antenna to the $i$-th receiver element.

For absolute comparison purposes between different scenarios, the capacity potential is evaluated via the eigen distribution of the MIMO links using the singular value decomposition of the channel transfer matrix $\mathbf{H}$, i.e.

$$
\mathbf{H}=\mathbf{U S V}^{H} \text {. }
$$

The unitary matrices $\mathbf{V}$ and $\mathbf{U}$ contain the right (input) and left (output) singular vectors of $\mathbf{H}$ respectively. $\mathbf{S}$ is a diagonal matrix with diagonal elements $s$, which are the singular values of $\mathbf{H}$.

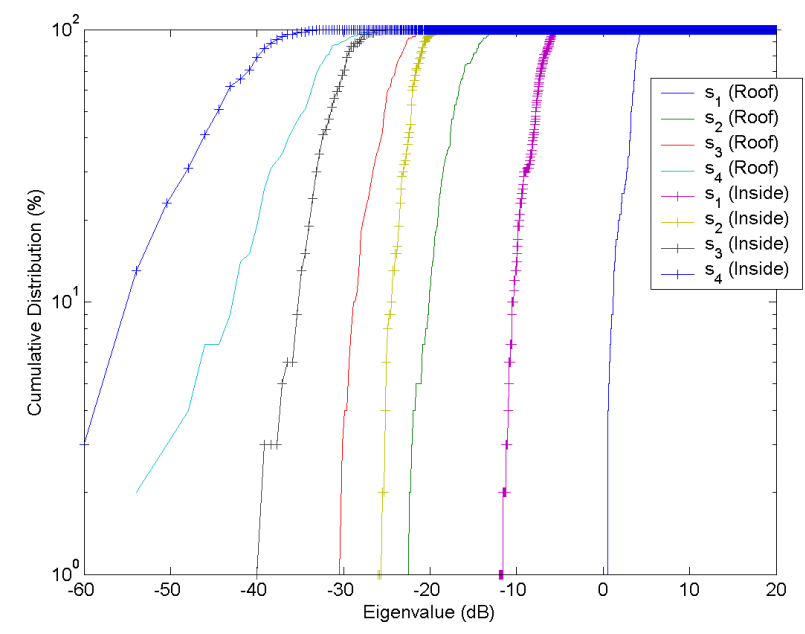

Fig. 4. Comparison of the eigenvalue distributions for a single data block from convoy measurements

The eigenvalue distributions of a single data section (100 samples) are given in figures 4 and 5, showing the roof array to roof array links (Roof) as well as the Roof to inside Laptop links (Inside). It follows from the example of the convoy measurement in figure 4 , that roof link $s_{1}$ is about about $10 \mathrm{~dB}$ higher than the inside link $s_{1}$, indicating a penetration loss of about 10dB. Furthermore the separation between the two highest eigenvalues $\left(s_{1}\right.$ and $\left.s_{2}\right)$ is about $20 \mathrm{~dB}$ for roof links, indicating very strong line-of-sight (LOS) conditions. For the inside links the separation is somewhat smaller at $12 \mathrm{~dB}$, as expected to possible local scattering inside the vehicle. The example from incident measurements in figure 5 shows more overall gain due to closer vehicle spacing. The roof and inside links now have comparable separation of dominant eigenvalues of about $12 \mathrm{~dB}$. This could be due to other traffic or other stationary objects such as road signs. Also the penetration loss 


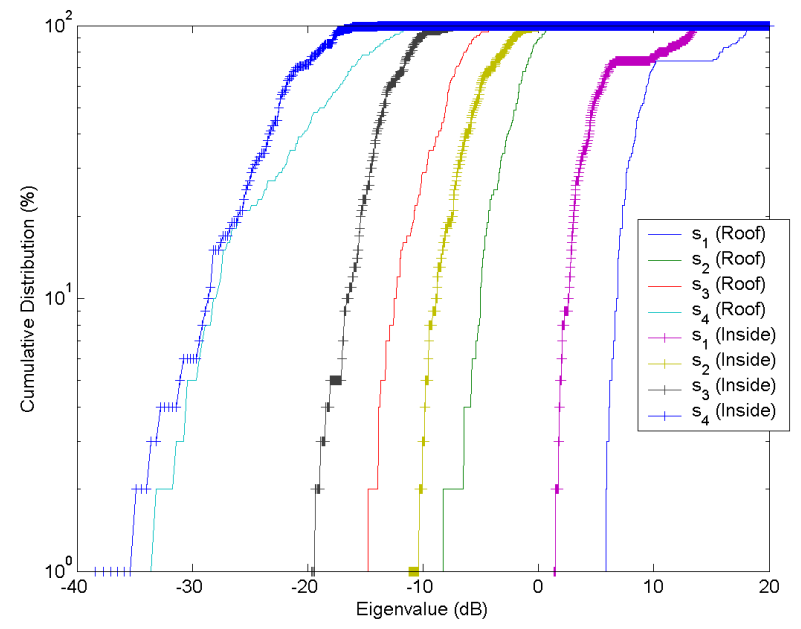

Fig. 5. Comparison of the eigenvalue distributions for a single data block from incident measurements

effect (difference of $s_{1}$ for roof and inside links) is drastically reduced to about $4 \mathrm{~dB}$. An explanation is that the lead vehicle in the convoy setup (having the inside Laptop array) had a large metallic platform/lift folded up inside and blocking the back door (which would be the LOS direction). In the Incident measurement the direct path is coming in at the side of the vehicle and in through a side window that was not blocked with metallic structures.

For relative comparison purposes between the different scenarios, we use the scattering Richness as defined in [8]. The Richness is based on the normalized channel matrix $\widetilde{\mathbf{H}}$ (mean gain $1(0 \mathrm{~dB})$ )

$$
R(n)=\sum_{j=1}^{N} \log _{2} \overline{\widetilde{s}_{j}^{2}}
$$

where $n$ is the order (max. 4 in our case) and $\widetilde{s_{j}}$ are the singular values of $\widetilde{\mathbf{H}}$. The Richness is a convenient measure for scattering strength and gain independent of SNR [8].

The richness is plotted in figures 6 and 7, which is based on mean eigenvalues over 10-11 sections of data (1000-1100 samples). In the case of convoy measurements the sample is taken well away from the bridges in an open area and for the incident measurements, the time window where the two vehicles are passing is considered. Results indicate a reasonable consistency in richness for the convoy measurements with a strong Rice factor due to a low fourth order, $\mathrm{R}(4)$. It also follows that from $\mathrm{R}(2)$ and onwards for the roof links, a degenerating richness is also exhibited (as expected from the $20 \mathrm{~dB}$ separation between the highest eigenvalues). Contrary for the inside links, the addition of the 2 nd eigne value provides $R(2)$ with marginal richness increase.

In the case of incident measurements, there is evidence of a rapid drop in richness with increased Rice factor before and after the passing point (between 10000 and 15000ms in figure 7 ), where there is a momentary point of high richness, possibly due to scattering from vehicles in between. As expected, it also follows that the richness is much more dynamic (specially for the higher orders) than in the convoy scenario.

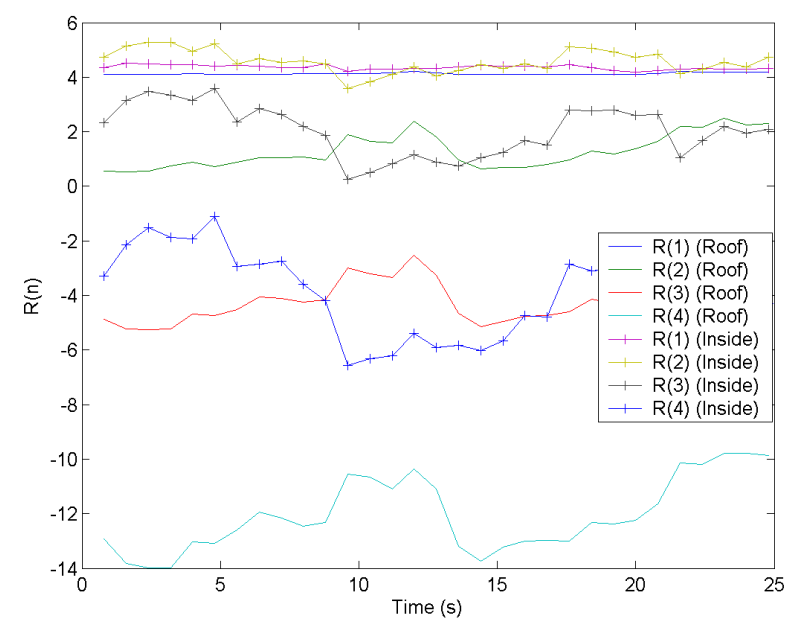

Fig. 6. Combined comparison of the richness plots for vehicles in convoy with the mobile both inside and outside vehicle 2

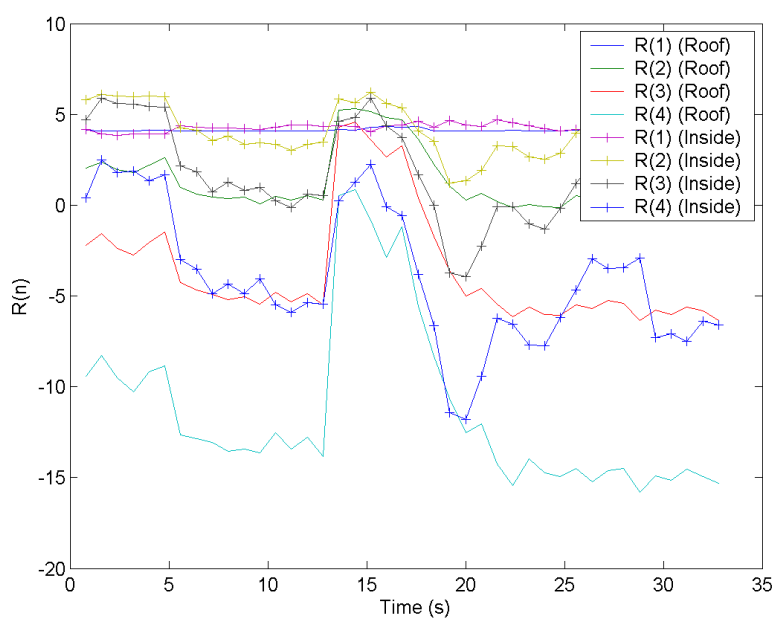

Fig. 7. Combined comparison of the richness plots for two incident vehicles with the mobile both inside and outside vehicle 2

\section{SCATTERING SCENARIO}

Using uniform linear array (ULA) antennas at both vehicles allows for simple dual-direction scanning to identify possible physical domain scattering clusters. In this paper we have chosen to apply the Capon method [9], which applies a Capon filter to determine the angle of arrival between $\pm 90^{\circ}$ on the broadside of a ULA. With the antennas applied in this measurement, there are inherent scan loss close to $\pm 90^{\circ}$, which creates limitations as the vehicles are passing.

The angular power distribution, $P(\theta)$, as a function of azimuth angle $\theta$ is derived from a Capon filter as: 


$$
P(\theta)=\frac{1}{\mathbf{a}^{H}(\theta) \mathbf{R}^{-1} \mathbf{a}(\theta)}
$$

Where the correlation matrix $\mathbf{R}$, is defined using $N$ narrowband sample spaces by:

$$
\mathbf{R}=\sum_{n=1}^{N} \mathbf{h}\left(t_{n}\right) \mathbf{h}^{H}\left(t_{n}\right)
$$

and $h$ is a $1 \times 4$ SIMO or MISO vector representing the physical channel link from one vehicle to the other. This can be done reciprocally given there are 4 branches at each end. The corresponding beamforming vector, $\mathbf{a}(\theta)$, is a four element phasor represented by $\left[1 e^{-j 2 \pi d \sin (\theta)} e^{-j 4 \pi d \sin (\theta)}\right.$ $\left.e^{-j 6 \pi d \sin (\theta)}\right]$, where $d$ is equal to 0.3 wavelengths. In this analysis, $N$ was set to 10 (zero padding) in order to compute a realizable inversion matrix, $\mathbf{R}^{-1}$.

The results of the Capon analysis for the Convoy measurement are presented in figure 8 versus time corresponding to the time period in figure 6 . The trace plots have approx. 20 $\mathrm{dB}$ dynamic range, from trace ridge to the noise floor. Due to scan loss issues, there is some inaccuracy in whether the angle of arrival is consistent, as would be expected with only minor differences due to interaction of other vehicles. The picture is unclear and therefore taking direct capon analysis of the actual array is not desirable for this purpose. Further to this, applying Capon analysis for the incident measurements in figure 9 shows that when the vehicles come to crossing in the middle of the plot, there is unclear behavior due to scan loss on the side of the array.

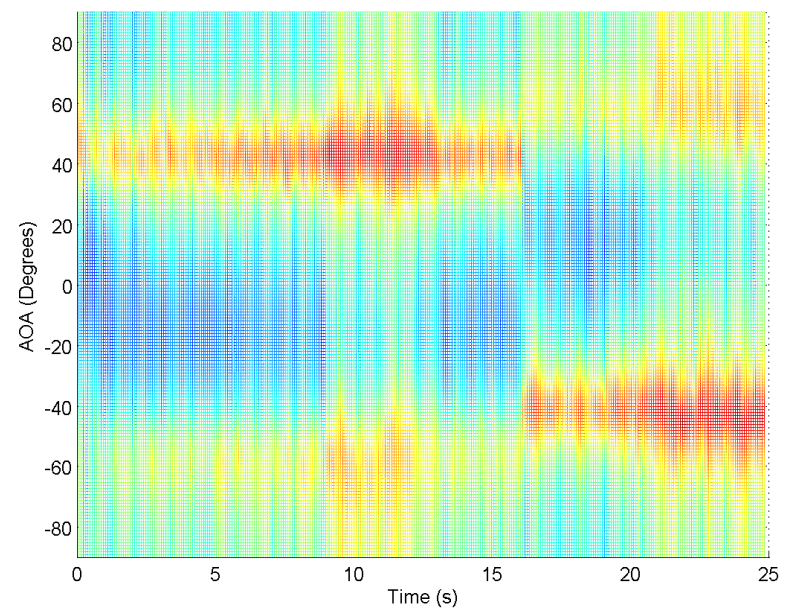

Fig. 8. Comparison of the Convoy AOA resolved for a $1 \mathrm{x} 4$ MISO link using the Capon method

To overcome the problem of scan loss in the array, we have proposed a synthetic array approach where 8 SISO link samples are taken at one point to represent a MISO or SIMO link. This is demonstrated in the incident case in figure 10 . It is the case, however, due to both vehicles moving that it is necessary to account for a phase error in the incident

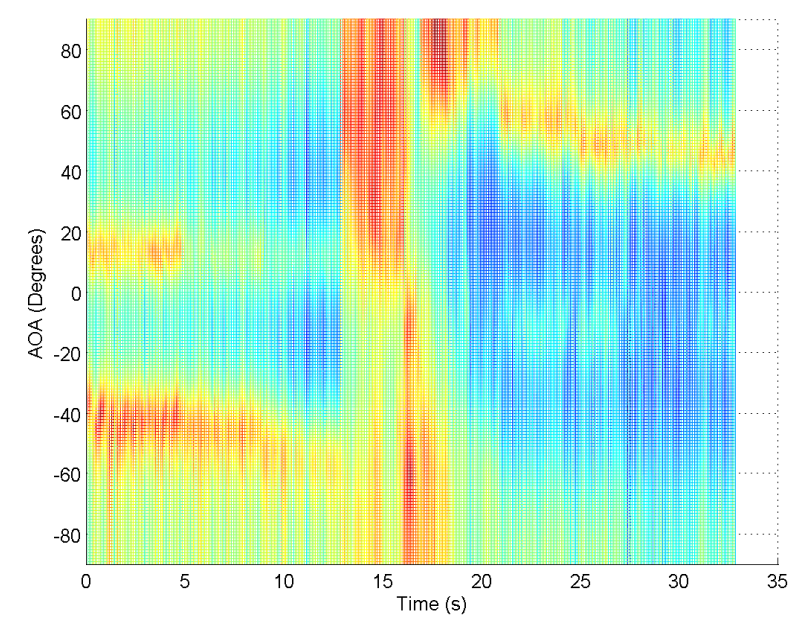

Fig. 9. Comparison of the Incident AOA resolved for a $1 \times 4$ MISO link using the Capon method

measurements. With a large distance between two vehicles compared to the effective antenna spacing, it is possible to approximate a phase correction equal to $2 \pi d \sin (\theta)$, where $\theta$ is shown in figure 10. This correction was not applied, however, in the convoy case. In this instance the 8 SISO links were following each other and treated as equivalent to stationary, co-phased elements, which they would be in free space. However, scattering from passing vehicles and other objects would disrupt this, so results could be analyzed to identify any rapid effect on angle of arrival due to scattering.

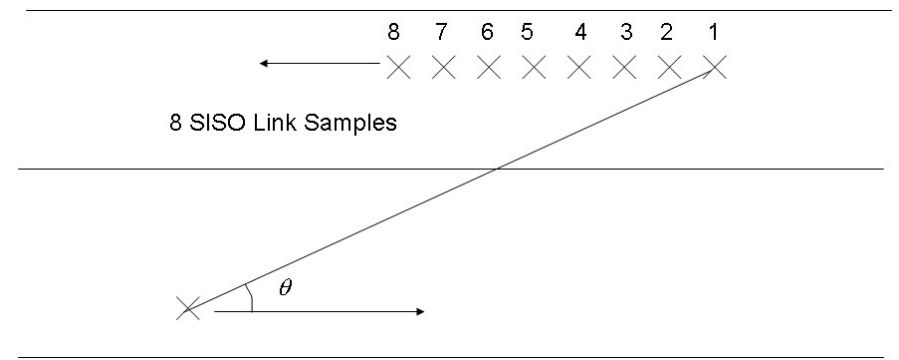

Fig. 10. Diagram explaining the synthetic array structure of the $1 \times 8$ SIMO link based on 8 SISO samples

Figure 11 shows a largely improved plot for the convoy case. In this instance, the center angle is at $\theta=90^{\circ}$ as the antennas are now in line with the vehicles. Also due to capon analysis there is a "mirror" effect in the beamforming so $\mathrm{AOA}$ at both $+90^{\circ}$ and $-90^{\circ}$ is shown where there is clear single line communication with little evidence of a secondary AOA. Therefore there is little evidence of effect from other vehicles that create any dual channel possibilities.

Figure 12 shows a much improved AOA in the incident case with the scan loss issues removed as the vehicles pass. There is an evident case here of a major angle of arrival 
starting at $-50^{\circ}$ with two other minor angles starting at $-10^{\circ}$ and $30^{\circ}$ possibly due to interaction from other vehicles. At crossing point there is generation of greater multipath, which would be expected from passing vehicles in between.

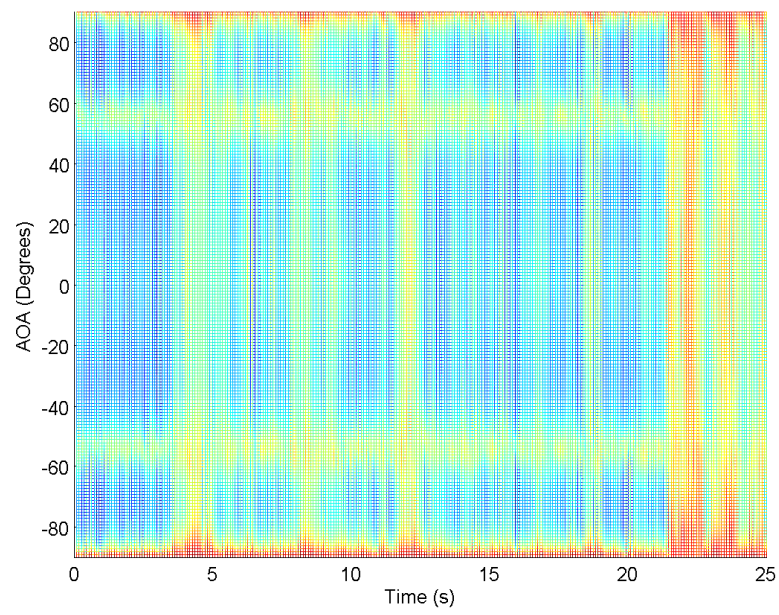

Fig. 11. Comparison of the Convoy AOA resolved for a $1 \mathrm{x} 8$ synthetic MISO link using the Capon method

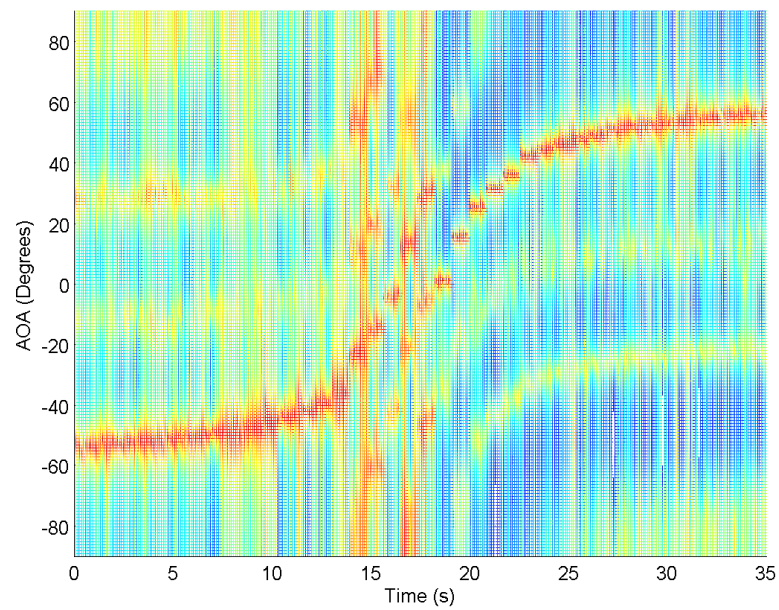

Fig. 12. Comparison of the Incident AOA resolved for a 1x8 synthetic MISO link using the Capon method

\section{CONCLUSION}

Even though the presented results are preliminary, we can identify that convoy style VTV MIMO communication can be facilitated in long haul transport applications. The higher modes seem very stable which suggest simple directing beam forming - and single higher order modulation data stream operation could be sufficient from a capacity point of view. However high speed operation caused Doppler stress, especially with incident traffic, both on phase and time synchronization loops, which can be foreseen to be a critical issue with respect to high order modulations and inter symbol interference.

\section{REFERENCES}

[1] G.J. Foschini and M.J. Gans, "On limits of wireless communications in a fading environment when using multiple antennas", Wireless Personal Communications, vol. 6, no. 3, pp. 311-335, March 1998.

[2] I.E. Telatar, "Capacity of Multi-antenna Gaussian Channels," AT\&T Bell Laboratories, Murray Hill, NJ, Technical note, 1996.

[3] J.B. Andersen, "Array gain and capacity for known random channels with multiple element arrays at both ends" in IEEE Journal on Selected Areas in Communication., vol. 18, pp. 2172-2178, November 2000.

[4] J.B. Andersen, R.G. Vaughan, "Channels, Propagation and Antennas for Mobile Communications", 2004, IEE, UK.

[5] J. B. Andersen, J. . Nielsen, G. F. Pedersen, K. Olesen, P. Eggers, E. H. Sørensen, S. Denno, "A 16 by 32 wideband multichannel sounder at 5 GHz for MIMO”, IEEE Antennas and Propagation Society Symposium, vol. 2, 20-25 June 2004, pp1263-1266.

[6] G. Acosta, K. Tokuda, M.A. Ingram, "Measured Joint Doppler-delay Power Profiles for Vehicle-to-vehicle Communications at $2.4 \mathrm{GHz}$ " IEEE Global Telecommunications Conference (GLOBECOM), vol. 6, 2004, pp3813-3817.

[7] W. A. Th. Kotterman, G. F. Pedersen, K. Olesen, P. Eggers, "Cable-less Measurement Setup for Wireless Handheld Terminals", IEEE International Symposium on Personal Indoor Mobile Communications, vol. 1, October 2001, pp. B112-116.

[8] J. Bach Andersen, "Propagation aspects of MIMO channel modelling" in "Space-Time Wireless Systems" (H. Blcskei et al.), Cambridge University Press, 2006.

[9] P.Stoica, R. Moses, "Introduction to Spectral Analysis", Prentice Hall, 1997, USA. 